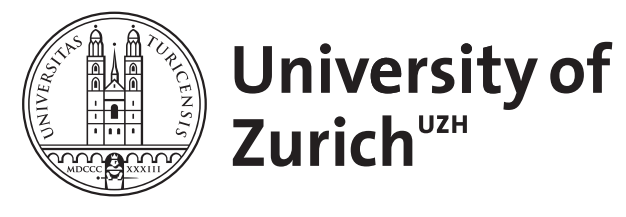

\title{
Molekular zielgerichtete Therapie
}

\author{
Stenner-Liewen, F ; Zippelius, A ; Pestalozzi, B C ; Knuth, A
}

\begin{abstract}
Until recently, cancer therapy was based on three modalities: surgery, radiotherapy, and cytostatic chemotherapy. In most instances treatment of solid tumors was a surgical domain. For patients with incomplete resection or relapse after surgery, radiotherapy and chemotherapy usually offered only partial response and mostly of limited duration. By the mid-1990s visions of antibody-based therapies, vaccination strategies, and even gene-specific therapies existed but seemed far from clinical practice. United States Federal Drug Administration approval of the humanized antibody rituximab (1997) and the tyrosine kinase inhibitor imatinib (2001) has changed perceptions of oncologic treatment. These drugs turned visions into reality and led the pharmaceutical industry, clinicians, and patients to new perspectives. This article gives an overview of the development of this fourth modality in cancer therapy, so-called targeted therapy.
\end{abstract}

DOI: https://doi.org/10.1007/s00104-006-1262-8

Other titles: Molecular targeted therapy

Posted at the Zurich Open Repository and Archive, University of Zurich

ZORA URL: https://doi.org/10.5167/uzh-156471

Journal Article

Published Version

Originally published at:

Stenner-Liewen, F; Zippelius, A; Pestalozzi, B C; Knuth, A (2006). Molekular zielgerichtete Therapie. Der Chirurg, 77(12):1118-1125.

DOI: https://doi.org/10.1007/s00104-006-1262-8 
Chirurg 2006 $\cdot 77: 1118-1125$

DOI 10.1007/s00104-006-1262-8

Online publiziert: 16. November 2006

(c) Springer Medizin Verlag 2006
F. Stenner-Liewen · A. Zippelius · B. C. Pestalozzi · A. Knuth

Klinik und Poliklinik für Onkologie, Universitätsspital Zürich

\section{Molekular zielgerichtete Therapie}

\begin{abstract}
Nach heutiger Auffassung stehen genetische Schäden am Anfang jeder Tumorerkrankung. Das Verständnis der Natur des jeweiligen Schadens ist der Schlüssel zu einer gezielten, sog. "targeted therapy". In einer "Ein Schaden führt zu einer Erkankung"-Situation, wie beispielsweise bei der chronischen myeloischen Leukämie (CML), wurde durch das zielgerichtete Medikament Imatinib (Gleevec ${ }^{\circ}$, Novartis) eine hocheffektive Therapie möglich. Hingegen ist für die Entstehung von soliden Tumoren meist die Akkumulation einer Reihe genetischer Fehler notwendig, sodass hier eine passgenaue Therapie ungleich schwieriger, vielleicht gar unmöglich erscheint. Doch auch hier zeichnen sich neue therapeutische Ansätze ab.
\end{abstract}

\section{Kinaseinhibitoren - „kleine Moleküle“}

Die rasanten Fortschritte der gegenwärtigen Tumortherapie mit der Entwicklung und Einführung einer Fülle neuer vielversprechender Medikamente sind nicht zufällig, plötzlich oder unerwartet, wie es dem verwunderten Laien erscheinen mag, sondern die Früchte langer und von vielen Rückschlägen gekennzeichneten globalen Forschungsbemühungen. Meilensteine auf dem Weg zur Entwicklung der sog. „small molecules" waren der Abschluss des humanen Genomprojektes (1990-2003) und die Analyse des humanen Kinomes [11]. Letztes beschäftigt sich mit der Entschlüsselung der Funktion von Proteinkinasen, einer zellbiologisch und pharmakologisch relevanten Enzymfamilie.
Proteinkinasen sind Enzyme, die durch ihre Aktivität Schlüsselfunktionen einfacher und komplexer Zellsysteme kontrollieren. Über 500 Kinasen wurden im menschlichen Genom identifiziert, sie stellen zwar nur 1,7\% aller Proteine, kontrollieren aber durch die Übertragung von Phosphatgruppen die Aktivität von etwa $30 \%$ aller Zellproteine. Sie geben Signale, die den Lebenszyklus, das Teilungsverhalten und die Interaktion der Zellen untereinander beeinflussen. Je nach Phosphorylierungsort unterscheidet man Threonin-, Serin- oder Tyrosinkinasen; Letztere sind die derzeit pharmazeutisch meistgenutzten.

Die Aktivität der Kinasen selbst unterliegt einer strengen Regulation. Einem Ein-Aus-Schalter vergleichbar befinden sich Kinasen meist in ihrer inaktiven Form. Im entscheidenden Moment, angestoßen durch beispielsweise ein hormonelles Signal, werden die Kinasen dann kurzfristig für die Übertragung von Phosphatgruppen aktiviert. Anschließend kehren sie in ihren ursprünglichen Ruhezustand zurück.

\section{— Überaktivität von Kinasen ist bei der Entstehung und Unterhaltung von Tumorerkrankungen von herausragender Bedeutung.}

Beispielhaft ist die ständige Aktivität der ABL-Kinase bei der CML. Hier werden, bedingt durch einen genetischen Schaden - die Translokation $\mathrm{t}(9 ; 22)$ (q34;q11) -, der die wachstumsfördernde ABL-Kinase permanent aktiviert, die mutationstragenden Zellen in eine unkontrollierte Teilung getrieben. Durch Hemmung der ATP-Bindungsstelle von ABL mittels Ima- tinib, einem ABL-Kinaseinhibitor, gelingt es, diesen Prozess zu stoppen und den fatalen Kreislauf von stetiger Zellteilung zu unterbinden [4].

Imatinib ist nicht allein ABL-Kinase spezifisch, sondern vermag auch c-Kit und den PDGF-R („platelet derived growth factor receptor") in ihrer Signalübertragung zu hemmen. Dadurch hat sich der klinische Einsatzbereich von Imatinib auf GIST-Tumoren (c-Kit) und das hypereosinophile Syndrom (PDGF-R) ausgeweitet.

Seit der klinischen Einführung von Gleevec $^{\circ}$ im Mai 2001 in den USA und dem großen klinischen Erfolg dieses Tyrosinkinaseinhibitors sind die Kinasen noch mehr in das Interesse der pharmazeutischen Industrie gerückt. Weitere Kinaseinhibitoren folgten. Ein Ende dieser Entwicklung ist nicht absehbar. Einige relevante Substanzen fasst $\bullet$ Tab. 1 zusammen. Zudem ist auch eine kontinuierliche Weiterentwicklung von bereits bewährten Kinaseinhibitoren möglich und Erfolg versprechend. So stehen für die Nachfolge von Imatinib heute schon 2 Substanzen, Dasatinib (Bristol-Myers-Squibb) und Nilotinib (Novartis), bereit [9, 22].

\section{Voraussetzungen für die Entwicklung epithelialer Tumoren}

Für die Entwicklung epithelialer Tumoren sind 2 Hauptvoraussetzungen notwendig.

\section{Kontinuierlich stimuliertes Zellwachs-} tum. Für Zellproliferation, Metastasierung und Invasion ist der epidermale Wachstumsfaktorrezeptors (EGF-R) hauptverantwortlich. Für dessen intrazelluläre Blockade stehen mit Gefitinib (Ires- 
Hier steht eine Anzeige.

第 Springer 


\begin{tabular}{|c|c|c|c|}
\hline Kinase & Nachgewiesene Störung & $\begin{array}{l}\text { Krankheitsbeispiele } \\
\text { (solide Tumoren) }\end{array}$ & Medikament \\
\hline c-Kit & Überexpression, Mutation & GIST, Glioblastom (GBM) & Imatinib Pazopanib \\
\hline Her2 & Überexpression, Mutation & $\begin{array}{l}\text { Mamma-, Bronchial- } \\
\text { karzinom (NSCLC) }\end{array}$ & Lapatinib \\
\hline PDGFRA & Mutation, Translokation & $\begin{array}{l}\text { Gastrointestinaler Stroma- } \\
\text { zelltumor (GIST) }\end{array}$ & Imatinib Pazopanib \\
\hline PDGFRB & Mutation, Translokation & Glioblastom (GBM) & Imatinib Pazopanib \\
\hline EGFR & Überexpression, Mutation & $\begin{array}{l}\text { Mammakarzinom, Ovarial- } \\
\text { karzinom, NSCLC, GBM }\end{array}$ & $\begin{array}{l}\text { Gefitinib Erlotinib } \\
\text { ZD6474 }\end{array}$ \\
\hline VEGF-R1,R2,R3 & Expression & $\begin{array}{l}\text { Neoangiogenese, unter- } \\
\text { schiedliche Tumoren }\end{array}$ & $\begin{array}{l}\text { Valatinib Pazopanib } \\
\text { Sorafenib }^{a} \\
\text { SunitinibaZZ6474 }\end{array}$ \\
\hline RET & Translokation, Mutation & Schilddrüsenkarzinome & ZD6474 \\
\hline
\end{tabular}

$\mathrm{sa}^{\oplus}$, AstraZeneca) und Erlotinib (Tarceva ${ }^{\oplus}$, Roche) bereits oral verfügbare Inhibitoren zur Verfügung, die ihre klinische Wirksamkeit bei einer Reihe von Tumoren, allen voran das nichtkleinzellige Bronchialkarzinom, gezeigt haben [19].

\section{Sicherstellung der Blutversorgung durch neue, „tumoreigene“ Gefäße. Das Ein-} sprossen neuer Blutgefäße, die sog. Neovaskularisation zum Unterhalt eines $\mathrm{Tu}$ mors ab einer kritischen Größe von 2$3 \mathrm{~mm}^{2}$ Durchmesser wurde durch Grundlagenforschung als kinaseregulierter Prozess verstanden. Die Hauptbeteiligten hier sind Rezeptoren der VEGF-Familie (vaskuläre endotheliale Wachstumsfaktorrezeptoren), membrangebundene Tyrosinkinasen, die Signale ins Innere der Zellen weitergeben. Deren Hemmung, zurzeit in klinischer Prüfung für einen dualen (EGF-R und VEGF-R) Kinaseinhibitor (ZD6474, AstraZeneca) beim nichtkleinzelligen Bronchialkarzinom, wird ein weiterer Schlüssel zu therapeutischen Erfolgen bei soliden Tumoren sein [5].

\section{Vorteile einer Therapie mit Kinaseinhibitoren und Entwicklungsmöglichkeiten}

Die besondere Eleganz einer Therapie mit Kinaseinhibitoren liegt in mehreren Eigenschaften begründet:

- Die Substanzen sind mit anderen

Therapieformen, seien es Antikörper-, Chemotherapien oder auch Vakzinierungsstrategien, kombinierbar.
- Kinaseinhibitoren sind bis heute relativ nebenwirkungsarm, die verursachten Therapiefolgen wie Hautveränderungen, Hypertonie und Durchfall sind für die Patienten zumeist akzeptabel.

- Sobald die pharmakologisch aktiven Substanzen, die sog. „small molecules", einmal definiert sind, lassen sie sich gut in großtechnischem Maßstab synthetisieren.

- Eine Weiterentwicklung dieser Substanzen wie verbesserte Galenik und steigerbare Wirksamkeit ist möglich.

- Kombinationen von Kinaseinhibitoren zur Unterbrechung von „parallelen“ Signalkaskaden sind denkbar. So genannte Multikinaseinhibitoren wie Sorafinib (Nexavar ${ }^{\oplus}$, Bayer) und Sunitinib (Sutent ${ }^{\oplus}$, Pfizer) zeigen bereits Erfolge bei bisher therapieresistenten Tumoren wie dem klarzelligen Nierenzellkarzinom.

Neben allem Optimismus sind aber auch Rückschläge zu verzeichnen. Der zweite kommerziell lancierte Kinaseinhibitor Gefitinib (Iressa ${ }^{\oplus}$ ) brachte keinen Vorteil gegenüber der Standardbehandlung beim nichtkleinzelligen Bronchialkarzinom. Ein ähnlicher Inhibitor, Erlotinib, zeigte bescheidene Erfolge, knapp 9\% Ansprechen bei einer vorbehandelten Patientengruppe mit fortgeschrittenen Bronchustumoren (Stadium IIIb/IV). Hingegen kann bei Nachweis einer EGF-R-Mutation nach einer anderen Studie ein Ansprechen auf Erlotininb von $75-100 \%$ erwartet werden [13].
Diese Unterschiede in ihrer Wirksamkeit zeigen Probleme und Herausforderungen zukünftiger Medikamentenentwicklung und Patientenselektion auf.

Die Therapie mittels Kinaseinhibitoren steht erst am Anfang einer Entwicklung, und wir dürfen gespannt sein auf den Benefit, den dieser Fortschritt Patienten mit gestörter Proteinkinasenaktivität bei so unterschiedlichen Erkrankungen wie Tumoren, Diabetes, Osteoporose und Entzündungsprozessen noch bringen wird.

Neben weiteren Anstrengungen zur Identifikation neuer Zielmoleküle bedarf es aber auch einer Verbesserung der Identifikation der Patienten, mittels molekularer Methoden, die von dieser „targeted therapy" profitieren. Zellbiologische, strukturbiochemische und röntgenkristallographische Daten werden dazu weitere Grundsteine legen.

\section{Monoklonale Antikörper}

Seit der Entwicklung der Hybridomtechnik 1975 können monoklonale Antikörper mit wählbarer Spezifität hergestellt werden. Zwanzig Jahre später gelang es, monoklonale Antikörper als Medikamente therapeutisch nutzbar zu machen. Dies wurde möglich durch eine sog. „Chimärisierung" der Antikörper. Hier wird die antigenbindende Stelle eines Mausantikörpers dem Gerüst eines menschlichen Antikörpermoleküls hinzugefügt. Solche „humanisierten“ Antikörper können zur „passiven“ Immuntherapie verwendet werden, da sie im Patienten keine primäre Immunantwort auslösen. Rituximab und Trastuzumab sind solche humanisierten Antikörper, die zur Behandlung des malignen Lymphoms bzw. des Mammakarzinoms eingesetzt werden. Therapeutische Antikörper lassen sich in Kombination mit konventionellen Zytostatika einsetzen.

\section{() „Humanisierte" Antikörper können zur „passiven“ Immuntherapie verwendet werden}

Die Onkologie ist nur eines der Fächer, das durch monoklonale Antikörper neue therapeutische Möglichkeiten erhalten hat. Die passive Immuntherapie mit industri- 
ell hergestellten monoklonalen Antikörpern hat nach Jahrzehnten der Skepsis den Durchbruch geschafft. Einen Überblick über Antikörper, die heute klinisch angewendet werden, gibt $\bullet$ Tab. 2.

Im Jahr 1975 haben Köhler und Milstein die Technik zur Herstellung monoklonaler Antikörper entwickelt und 1984 für diese Entdeckung den Nobelpreis erhalten. Wenn man eine Maus mit einem Antigen immunisiert und danach ihre lienalen Lymphozyten mit einer antikörpersekretierenden Plasmazelllinie zu einem Hybridom fusioniert, lassen sich unter den Hybridomen Klone isolieren, die Antikörper sekretieren, die spezifisch gegen das zur Immunisierung verwendete Antigen gerichtet sind. So kann man monoklonale Antikörper mit einer wählbaren Spezifität herstellen. Diese Technik führte zunächst zu großen Fortschritten in der biologischen Forschung und in der Diagnostik. Die therapeutische Verwendung ließ aber auf sich warten [1]. Versuche von Levy mit antiidiotypischen Antikörpern (individuell maßgeschneiderte Antikörper gegen antikörperbildende Lymphome) waren aufwendig und nur begrenzt wirksam. Auch konnten die Mausantikörper nur über kurze Zeit therapeutisch eingesetzt werden. Nach wenigen Monaten kam es zur Bildung von HAMA („humananti-mouse antibodies") und allergischen Reaktionen. In den 1990er-Jahren entwickelte die Firma IDEC Pharmaceuticals in Kalifornien die Technik der „Humanisierung“ von Antikörpern. Dabei wurde die antigenbindende variable Region des monoklonalen Mausantikörpers auf das Gerüst eines menschlichen IgG-Antikörpers gesetzt. Solche „humanisierten“ oder „chimären“ Antikörper werden vom Immunsystem in der Regel nicht mehr als fremd erkannt. Zudem kann die humane Effektordomäne im „Gerüst“ dieser Antikörper besser mit dem menschlichen Immunsystem interagieren und so zur therapeutischen Wirksamkeit beitragen. Der erste klinisch wirksame chimäre Antikörper ist Rituximab (Mabthera ${ }^{\varpi}$ ), der in den USA im Jahr 1997 zugelassen wurde.

\section{Rituximab}

Rituximab wurde hergestellt aus dem monoklonalen Mausantikörper Ibritu-

Chirurg 2006 ·77:1118-1125 DOI 10.1007/s00104-006-1262-8

(c) Springer Medizin Verlag 2006

F. Stenner-Liewen · A. Zippelius · B. C. Pestalozzi · A. Knuth Molekular zielgerichtete Therapie

Zusammenfassung

Bis vor knapp 10 Jahren stützte sich die Tumortherapie auf 3 Säulen: die Chirurgie, die Strahlentherapie und die zytostatische Chemotherapie. Eine definitive Heilung im Bereich der soliden Tumoren versprach meist nur eine vollständige Entfernung des Tumors durch den Chirurgen. Radiotherapeuten und Onkologen konnten nur einem kleinen Teil ihrer Patienten langfristig helfen. Antikörpertherapien, Antitumorvakzinierungen oder gar genspezifische, individualisierte Therapieformen existierten zwar als Visionen, diese schienen Mitte der 90er-Jahre von einer klinischer Anwendung noch weit entfernt. Mit der Einführung des Antikörpers Rituximab (1997) und des Tyrosinkinaseinhibitors Imatinib (2001) in die klinische Praxis kamen

\section{Molecular targeted therapy}

\section{Abstract}

Until recently, cancer therapy was based on three modalities: surgery, radiotherapy, and cytostatic chemotherapy. In most instances treatment of solid tumors was a surgical domain. For patients with incomplete resection or relapse after surgery, radiotherapy and chemotherapy usually offered only partial response and mostly of limited duration. By the mid-1990s visions of antibody-based therapies, vaccination strategies, and even gene-specific therapies existed but seemed far from clinical practice. United States Federal Drug Administration approval of the hu-
2 neuartige Substanzen auf den Markt, die Denken und Vorstellungen in der Onkologie grundsätzlich veränderten. Diese Therapeutika ließen aus Visionen Realitäten werden, die der Pharmaindustrie, den Klinikern und Patienten neue Perspektiven bezüglich Machbarkeit und kommender Möglichkeiten im Bereich der Tumortherapie eröffnet haben. Im Folgenden soll ein Überblick über die Entwicklung der 4. Säule der Tumortherapie, der sog. „targeted therapy“, gegeben werden.

\section{Schlüsselwörter}

Therapeutische Antikörper · Kinaseinhibitoren · Tumorvakzinierung · Rituximab · Imatinib

manized antibody rituximab (1997) and the tyrosine kinase inhibitor imatinib (2001) has changed perceptions of oncologic treatment. These drugs turned visions into reality and led the pharmaceutical industry, clinicians, and patients to new perspectives. This article gives an overview of the development of this fourth modality in cancer therapy, so-called targeted therapy.

\section{Keywords}

Small molecules - Therapeutic antibodies · Tumor vaccination $\cdot$ Rituximab $\cdot$ Imatinib 


\begin{tabular}{|lll|}
\hline Tab. 2 & Therapeutische & Antikörper \\
\hline Antikörper & Antigen & Krankheit \\
Rituximab & CD20 & $\begin{array}{l}\text { Non-Hodgkin- } \\
\text { Lymphom }\end{array}$ \\
Alemtuzumab & CD52 & CLL \\
\hline $\begin{array}{l}\text { Gemtuzumab } \\
\text { Trastuzumab }\end{array}$ & HER2 & AML \\
\hline Cetuximab & EGFR & $\begin{array}{l}\text { Mamma- } \\
\text { karzinom }\end{array}$ \\
\hline Bevacizumab & VEGF & Kolonkarzinom \\
\hline
\end{tabular}

Tab. 3 Rituximab (Mabthera ${ }^{\circ}$ ) Mechanismus: Bindung an CD20, Zelltod durch ADCC, CDC und/oder Apoptose

Wirksam in Kombination mit Chemotherapie bei allen B-Zell-Non-Hodgkin-Lymphomen (follikuläres Lymphom, großzelliges Lymphom, Mantelzelllymphom, BurkittLymphom, posttransplantäre Lymphome, HIV-assoziiertes Lymphom u. a.)

Tab. 4 HER2-Status und Trastuzumab

(Herceptin $\left.{ }^{\circledast}\right)$

HER2/neu/c-erbB-2 ist ein Membranrezeptor

Bei 25\% der Mammakarzinome ist HER2 überexprimiert (Immunhistochemie ++ oder +++$)$

FISH (Fluoreszenz-in-situ-Hybridisierung) zur Bestätigung der Genamplifikation

Trastuzumab (Herceptin ${ }^{\circ}$ ) ist ein humanisierter monoklonaler Antikörper Trastuzumabmonotherapie Trastuzumab+Chemotherapie (Paclitaxel, Docetaxel, Vinorelbine) in der palliativen Behandlung des Mammakarzinoms

Trastuzumab+Chemotherapie in der adjuvanten Behandlung

momab gegen das $\mathrm{CD}_{20} \mathrm{O}$-Rezeptormolekül, das auf aktivierten und neoplastischen B-Lymphozyten vorkommt. Das Antikörpergerüst von Rituximab besteht aus einem menschlichen IgG1-Molekül. Zwar ist bekannt, wie Rituximab an die B-Lymphozyten bindet, hingegen wissen wir nur sehr ungenau, wie diese Bindung zum Zelltod führt (• Tab. 3). Dieser kann vermittelt werden durch ADCC (,antibody-dependent cell cytotoxicity") z. B. über Makrophagen, via Komplement („,complement dependent cytotoxicity", CDC), via Apoptose (programmierter suizidaler Zelltod) oder durch eine Kombination dieser und anderer Mechanismen [1].

Klinische Studien haben gezeigt, dass Rituximab bei quasi allen B-Zell-Lymphomen wirksam ist. Bei den follikulären
Non-Hodgkin-Lymphomen ist Rituximab sowohl in Monotherapie als auch in Kombination mit Zytostatika wirksam. Beim diffus großzelligen Lymphom und anderen aggressiven B-Zell-Lymphomen (z. B. Mantelzelllymphom, Burkitt-Lymphom) ist es in Kombination mit Zytostatika wirksam. Eine europäische Multicenterstudie hat nachgewiesen, dass die Kombination von Rituximab mit CHOP (Cyclophosphamid, Doxorubicin, Vincristin, Prednison) der alleinigen Chemotherapie mit CHOP in der Behandlung des diffus großzelligen B-Zell-Lymphoms überlegen ist [2].

\section{Trastuzumab}

Trastuzumab (Herceptin $\left.{ }^{\circ}\right)$ ist gegen den Membranrezeptor HER2 (, human epithelial receptor 2") auf Mammakarzinomzellen gerichtet. Beim Menschen sind 4 Typen dieser epithelialen Rezeptoren bekannt (HER1, HER2, HER 3 , HER4; - Tab.4). Bei Stimulation durch extrazelluläre Liganden (z. B. Wachstumsfaktoren wie EGF, ,epithelial growth factor") wird ein Wachstumssignal nach intrazellulär durch die Tyrosinkinaseaktivität des intrazellulären Teils des Rezeptors weitergegeben. Es ist wahrscheinlich, dass die maligne Entartung gewisser Mammakarzinome dadurch zustande kommt, dass HER2 mutiert oder überexprimiert wird und so von der Membran aus dem Zellinneren ständig Wachstumsbedarf signalisiert wird ohne extrazelluläre Kontrolle. Bei einem Viertel der Mammakarzinome ist eine Überexpression des HER2 auf der Tumorzelle vorhanden. Nur bei diesen Karzinomen ist eine Therapie mit Trastuzumab sinnvoll. Zum Nachweis der HER2-Überexpression wird in den meisten Labors die Immunhistochemie verwendet, die eine semiquantitative Einschätzung der Rezeptordichte ergibt. Nur ein ++- oder +++-Resultat gilt als „positiv". Insbesondere bei einem ++Resultat sollte zusätzlich die Genamplifikation mittels FISH-Test (Fluoreszenzin-situ-Hybridisierung) bestimmt werden [17]. Diese korreliert besser mit dem Tumoransprechen auf Trastuzumab als der immunhistochemische Nachweis. Heute wird der HER2-Rezeptor routinemäBig nach der Primäroperation des Mam- makarzinoms bestimmt - ähnlich wie die Östrogen- und Progesteronrezeptoren.

\section{- Trastuzumab ist in der Behandlung des fortgeschrittenen Mammakarzinoms mit HER2- Überexpression wirksam.}

Nach zytostatischer Vorbehandlung erreicht die Monotherapie mit Trastuzumab Ansprechraten von um 15\%. Die Kombination von Trastuzumab mit Chemotherapie ist der alleinigen gleichen Chemotherapie bezüglich Ansprechrate, aber auch im progressionsfreien Überleben und im Gesamtüberleben überlegen [21]. Das ist für Paclitaxel und kürzlich auch für Docetaxel in großen randomisierten Phase-III-Studien belegt worden. Wegen erhöhter Kardiotoxizität sollte Trastuzumab nicht mit Anthrazyklinen kombiniert werden. Der größte Erfolg dieses Antikörpers kam im Jahre 2005 mit dem Nachweis in mehreren großen randomisierten Studien, dass krankheitsfreies Überleben und Gesamtüberleben durch eine adjuvante Behandlung mit Trastuzumab über 1 Jahr hochsignifikant verbessert werden $[14,16]$.

\section{Hämatologische Neoplasien}

Neben Rituximab wurden weitere monoklonale Antikörper gegen Antigene auf hämatologischen Tumorzellen entwickelt [1]. Alemtuzumab (MabCampath ${ }^{\circ}$ ) ist ein humanisierter Rattenantikörper gegen $\mathrm{CD}_{52}$, ein Membranglykoprotein, das auf allen Lymphozyten und in hoher Konzentration auf malignen Lymphozyten exprimiert wird. Die Radioimmunkonjugate von anti-CD2o-Antikörper mit einem Isotop ${ }^{90}$ Yttrium-Ibritumomab Tiutexan (Zevalin ${ }^{\circ}$ ) und ${ }^{131}$ Iod-Toxitumomab (Bexxar $\left.{ }^{\circledR}\right)$. werden bei Rezidivlymphomen eingesetzt.

Gemtuzumab ist ein monoklonaler Mausantikörper gegen das $\mathrm{CD}_{33}$-Antigen, das auf $80 \%$ der leukämischen Blasten bei akuter myeloischer Leukämie (AML) zu finden ist. Gemtuzumab-Ozogamicin (GO; Mylotarg ${ }^{\circ}$ ) ist ein Immunotoxin, in dem der Antikörper Gemtuzumab mit dem Toxin Calicheamycin konjugiert ist. 


\section{Solide Tumoren}

Im Jahr 2003 wurde Cetuximab (Erbitux ${ }^{\circ}$ ), ein "humanisierter" monoklonaler Antikörper, zur Behandlung des fortgeschrittenen Kolorektalkarzinoms in Kombination mit dem Zytostatikum Irinotecan bei Resistenz auf Irinotecan allein zugelassen. Er ist gegen den EGFR (,epidermal growth factor receptor" oder HER1) gerichtet.

Bevavizumab, ein ebenfalls humanisierter monoklonaler Antikörper, ist gegen VEGF (,vascular endothelial growth factor") gerichtet. VEGF, ein löslicher Faktor, vermittelt die Neubildung von Gefäßen, ohne die Tumoren und Metastasen nicht über eine Größe von $2 \mathrm{~mm}$ hinaus wachsen können. Vielversprechende Resultate einer großen randomisierten Studie mit Bevacizumab wurden 2003 präsentiert [6]. Dabei zeigten sich sowohl ein besseres Tumoransprechen als auch ein längeres Überleben im Studienarm mit der Kombination von Chemotherapie mit
Bevacizumab gegenüber alleiniger Chemotherapie mit Irinotecan, Fluorouracil und Leukovorin (IFL). Seltene Nebenwirkungen von Bevacizumab waren Blutungen sowie die Entwicklung einer arteriellen Hypertonie. Dies ist die erste klinische Studie mit gesichertem Vorteil einer Antiangiogenesetherapie. Es ist anzunehmen, dass Bevacizumab nicht nur beim Kolorektalkarzinom, sondern auch bei anderen soliden Tumoren wirksam sein wird.

Neben den erwähnten, in der Klinik eingesetzten Antikörpern wartet eine ganze Reihe weiterer Antikörper in der „Pipeline“.

Es gibt also 2 Kategorien von Medikamenten, die eine gezieltere („targeted“) und relativ gut verträgliche onkologische Therapie ermöglichen: Antikörper und „small molecules“. Diese Medikamente sind durchaus miteinander und auch mit der konventionellen zytostatischen Therapie kombinierbar. So haben sich der Onkologie in den letzten Jahren neue thera- peutische Möglichkeiten eröffnet, die wir für unsere Patienten einsetzen können.

\section{Tumorvakzinierung}

Die Vorstellung, dass das Immunsystem in der Lage ist, die Entstehung und den Verlauf maligner Erkrankungen zu beeinflussen, hat im vergangenen Jahrhundert wiederholt Auftrieb und Ernüchterung erfahren. Paul Ehrlich stellte bereits 1909 die These auf, dass „die Zerstörung von spontan auftretenden Tumoren eine der Aufgaben des Immunsystems" sei. In den 5oer-Jahren postulierten Burnet und Thomas, dass das Immunsystem den Körper überwacht und Tumorzellen zerstört, noch bevor diese klinisch apparent werden. Neuere experimentelle Daten belegen eindeutig, dass das Immunsystem eine wichtige Rolle in der Immunüberwachung spielt. Hauptrepräsentanten der zellulären Immunantwort sind dabei $\mathrm{CD}_{4}{ }^{+}$- und $\mathrm{CD} 8^{+}$-T-Zell-Subpopulationen. Während $\mathrm{CD}^{+}$-zytotoxische $\mathrm{T}$ -

\section{Hier steht eine Anzeige.}

\section{Springer}


Zellen nach Antigenerkennung die spezifische Zerstörung antigenexprimierender Zellen vermitteln können, erfüllen $\mathrm{CD}_{4}{ }^{+}-$ T-Zellen v. a. eine „Helferfunktion“, indem sie zur Initiierung und Verstärkung der CD8 ${ }^{+}$-T-Zell-Antwort beitragen.

Die molekulare Charakterisierung von Tumorantigenen bildet die Grundlage für die Entwicklung spezifischer, aktiver Immuntherapieverfahren bei malignen Erkrankungen [23]. Tumorantigene werden nach Gewebsexpression, Genfunktion oder Entstehung in mehrere Untergruppen eingeteilt. Ideale Tumorantigene sollten im Tumor möglichst spezifisch und homogen exprimiert sein, dagegen sollten sie in normalen Geweben nicht bzw. nur möglichst eingeschränkt vorkommen. Eine für aktive Immuntherapie interessante Kategorie von Tumorantigenen stellen „Cancer-Testis-“ (CT-)Antigene dar. Diese werden physiologisch lediglich von Keimzellen des Testis, der fötalen Ovarien sowie der Plazenta exprimiert [3, 2o]. Ihre „aberrante“ Expression in Tumoren verschiedenster Herkunft wird als Aktivierung des stillgelegten „Keimzellprogrammes" in somatischen Zellen verstanden. In die Gruppe der CT-Antigene gehören die Antigene MAGE, BAGE, GAGE und NY-ESO-1. Das Antigen NYESO-1 scheint gegenwärtig das CT-Antigen mit der höchsten Immunogenität zu sein. Viele Patienten mit v. a. fortgeschrittenen Tumoren zeigen kombinierte humorale und T-Zell-vermittelte (sog. integrierte) Immunantworten gegen NYESO-1 [7].

Daneben sollen hier nur noch kurz Differenzierungsantigene erwähnt werden, die in einem ausdifferenzierten Zelltyp und dem entsprechenden Tumortyp, wie etwa in Melanozyten und im malignen Melanom, exprimiert sind. Die Expression dieser Antigene ist meist hoch, anders als bei CT-Antigenen geht sie mit zunehmendem Entdifferenzierungsgrad der Zellen bei Progression der Erkrankung zurück. Proteine wie Tyrosinase, Glykoprotein 100 (gp1oo) und Melan-A/ MART-1 sind an der Biosynthese von Melanin in Melanosomen beteiligt. Eines der bedeutendsten neuen Differenzierungsantigene der Brustdrüse ist NY-BR1. Dies ist auf Mammakarzinomzellen in hohem Maß exprimiert und hat das Potenzial für ein spezifisches „Target“ für Immuninterventionen [24].

\section{Peptidvakzinierung}

Zahlreiche klinische Studien zur aktiven, T-Zell-basierten Immuntherapie sind in den letzten Jahren durchgeführt worden [12]. Im Fall der spezifischen Impfung kann dabei das betreffende Tumorantigen in verschiedenen Formen eingesetzt werden. Vakzinierungen mit Peptidantigenen, die vergleichsweise leicht und kostengünstig zu synthetisieren sind, wurden in einer großen Anzahl von klinischen Phase-I/II-Studien bei Patienten mit soliden Tumoren getestet. Wiederholt konnte gezeigt werden, dass eine Peptidvakzinierung eine tumorantigenspezifische TZell-Antwort induzieren kann. Eine angemessene Immunreaktion entsteht regelmäßig allerdings erst unter gleichzeitiger Applikation von Adjuvanzien, die unter anderem die Auslösung einer inflammatorischen Reaktion, die Aktivierung spezifischer T-Zellen oder die verbesserte Präsentation des Antigens bewirken können. Die unerwünschten Wirkungen beschränken sich auf Lokalreaktionen an den Injektionsstellen und selten Temperaturerhöhungen mit grippeähnlichen Symptomen. Da die T-Zell-Erkennung von Peptiden auf bestimmte HLAMoleküle restringiert ist, können Peptidvakzinierungen im Allgemeinen nur für Patienten mit einem bestimmten HLATyp eingesetzt werden. Die meisten Impfungen werden mit $\mathrm{HLA}^{\star}$ O2O1-restringierten Peptiden durchgeführt, da dieser Genotyp mit einer Verbreitung von etwa $50 \%$ der häufigste der weißen, westlichen Bevölkerung ist. Andere Formen von Tumorantigenen, die einerseits potenzielle HLA-Klasse-I- und -II-restringierte TZell-Epitope beinhalten und andererseits bei allen Patienten unabhängig vom HLATyp eingesetzt werden können, sind rekombinante Tumorproteine oder für Tumorantigene kodierende DNA/RNA, die „nackt“ oder in viralen Vektoren verpackt für die Transfektion von antigenpräsentierenden Zellen verwendet werden [8].

Besonders ermutigende immunologische und klinische Ergebnisse wurden nach Vakzinierung mit Peptidantigenen erzielt, die sich von NY-ESO-1 ableiten.
Es konnten einige partielle und wenige komplette Remissionen beobachtet werden, die im Gegensatz zu chemotherapieinduzierten Remissionen oft sehr beständig sind.

Es ist eine Aufgabe der Zukunft diese vielversprechenden neuen Therapiemodalitäten in geeigneter Weise in die heutige onkologische Praxis zu integrieren.

\section{Potenzial der Immuntherapie}

Die umfassende Charakterisierung der DC als die wichtigsten und effizientesten antigenpräsentierenden Zellen haben zu einer Vielzahl von klinischen Studien geführt [18]. Dabei werden diese Zellen aus $\mathrm{CD}_{34} 4^{+}-$bzw. $\mathrm{CD}_{14}{ }^{+}$-Zellen im Blut gewonnen, ex vivo mit definierten Tumorantigenen (Peptid, Protein, RNA, DNA) oder undefinierten Tumorzellpräparationen (Lysat, Tumor-RNA) beladen und dem Patienten injiziert. Obwohl meist spezifische Immunantworten induziert werden können, sind die bisherigen klinischen Resultate nicht eindeutig. Die Herstellung von DC ex vivo ist darüber hinaus aufwendig und erfordert GMP(„good manufactering practice-“)Bedingungen.

Parallel mit der Entwicklung neuer Immuntherapieansätze werden hochempfindliche Methoden des Monitorings einer antigenspezifischen Immunantwort entwickelt. Als klinisch-immunologischer Indikator einer signifikanten Immunantwort ist die „delayed-type hypersensitivity-“ (DTH-)Reaktion definiert, eine Immunreaktion vom verzögerten Typ, die 48 h nach erneuter Antigeninjektion in ihrer Qualität und Stärke beurteilt wird und mit der Induktion spezifischer T-Zell-Antwort korreliert werden kann. Der Einsatz neuer Immunisierungsstrategien mit "nackter" RNA/DN ist derzeit in Entwicklung und verspricht eine neue Dimension in der Immuntherapie maligner Tumoren [15].

Insgesamt zeigen erste Studien der aktiven Immuntherapie ermutigende Ergebnisse mit klinischen Remissionen bei einzelnen Patienten, bemerkenswert langen progressionsfreien Intervallen und objektiv messbaren immunologischen Parametern. Die klinische Wirksamkeit in der Onkologie ist derzeit allerdings limitiert. 
Die optimale Induktion und Aufrechterhaltung einer antitumoralen Immunantwort sowie die Überwindung von Toleranzmechanismen sind Gegenstand aktueller klinisch-immunologischer Forschung. Die ersten Erfolge der präventiven Immunisierung z. B. gegen viral induzierte Karzinome der Zervix unterstreichen das Potenzial einer zellulären Immuntherapie und lassen die Hoffnung $\mathrm{zu}$, dass einige Impfstoffe demnächst zur Verfügung stehen werden [10].

\section{Fazit für die Praxis}

Antikörpertherapien, Antitumorvakzinierungen oder genspezifische, individualisierte Therapieformen stellen heute die 4. Säule der Tumortherapie, der sog. „targeted therapy", dar. Mit der Einführung des Antikörpers Rituximab (1997) und des Tyrosinkinaseinhibitors Imatinib (2001) in die klinische Praxis kamen 2 neuartige Substanzen auf den Markt, die Denken und Vorstellungen in der Onkologie grundsätzlich veränderten. Auch die Tumorvakzinierung bietet neue Therapieansätze - auch im präventiven Bereich -, deren Potenzial noch bei weitem nicht ausgeschöpft ist.

\section{Korrespondierender Autor \\ Dr. F. Stenner-Liewen \\ Klinik und Poliklinik für Onkologie, \\ Universitätsspital Zürich, \\ Rämistraße 100, 8091 Zürich \\ Frank.Stenner@usz.ch}

Interessenkonflikt. Es besteht kein Interessenkonflikt. Der korrespondierende Autor versichert, dass keine Verbindungen mit einer Firma, deren Produkt in dem Artikel genannt ist, oder einer Firma, die ein Konkurrenzprodukt vertreibt, bestehen. Die Präsentation des Themas ist unabhängig und die Darstellung der Inhalte produktneutral.

\section{Literatur}

1. Byrd JC (2003) Monoclonal antibody therapy for hematologic malignancies. Sem Oncol 30: 421

2. Coiffier B, Lepage E, Brière J et al. (2002) CHOP chemotherapy plus rituximab compared with $\mathrm{CHOP}$ alone in elderly patients with diffuse large-B-cell lymphoma. N Engl J Med 326: 235

3. Gnjatic S, Nishikawa $H$, Jungbluth $A A$ et al. (2006) NY-ESO-1: review of an immunogenic tumor antigen. Adv Cancer Res 95: 1

4. Hehlmann R, Berger U, Hochhaus A (2005) Chronic myeloid leukemia: a model for oncology. Ann Hematol. 84: 487
5. Holden SN, Eckhardt SG, Basser R et al. (2005) Clinical evaluation of ZD6474, an orally active inhibitor of VEGF and EGF receptor signaling, in patients with solid, malignant tumors. Ann Oncol 16: 1391

6. Hurwitz H, Fehrenbacher L, Novotny W et al. (2004) Bevacizumab plus Irinotecan, Fluorouracil, and Leucovorin for metastatic colorectal cancer. N Engl J Med 350: 2335

7. Jager $E$, Jager D, Karbach J et al. (2000) Identification of NY-ESO-1 epitopes presented by human histocompatibility antigen (HLA)-DRB4*01010103 and recognized by CD4(+) T lymphocytes of patients with NY-ESO-1-expressing melanoma. J Exp Med 21;191(4): 625

8. Jager E, Karbach J, Gnjatic S et al. (2006) Recombinant vaccinia/fowlpox NY-ESO-1 vaccines induce both humoral and cellular NY-ESO-1-specific immune responses in cancer patients. PNAS 103: 14453

9. Kantarjian H, Giles F, Wunderle L et al. (2006) Nilotinib in imatinib-resistant CML and Philadelphia chromosome-positive ALL. N Engl J Med 354: 2542

10. Koutsky LA, Ault KA, Wheeler CM et al. (2006) A controlled trial of a human papillomavirus type 16 vaccine. N Engl J Med 347: 1645

11. Manning G, Whyte DB, Martinez R et al. (2002) The protein kinase complement of the human genome. Science 298: 1912

12. Nicholaou T, Ebert L, Davis ID et al. (2006) Directions in the immune targeting of cancer: lessons learned from the cancer-testis Ag NY-ESO-1. Immunol Cell Biol 84: 303

13. Paz-Ares L et al. (2006) A prospective phase II trial of erlotinib in advanced non-small cell lung cancer (NSCLC) patients with mutations in the tyrosine kinase domain of the epidermal growth factor receptor (EGFR). J Clin Oncol, 2006 ASCO Annual Meeting Proceedings Vol 24, No. 18S: 7020

14. Piccart-Gebhart MJ, Procter M, Leyland-Jones B et al. (2005) Trastuzumab after adjuvant chemotherapy in HER2-positive breast cancer. N Engl J Med 353: 1659

15. Rammensee HG (2006) Some considerations on the use of peptides and mRNA for therapeutic vaccination against cancer. Immunol Cell Biol 84: 290

16. Romond EH, Perez EA, Bryant J et al. (2005) Trastuzumab plus adjuvant chemotherapy for operable HER2-positive breast cancer. N Engl J Med 353. 1673

17. Ross JS, Fletcher JA, Linette GP et al. (2003) The HER-2/neu gene and protein in breast cancer 2003: biomarker and target of therapy. Oncologist 8: 307

18. Schuler G, Schuler-Thurner B, Steinman RM (2003) The use of dendritic cells in cancer immunotherapy. Curr Opin Immunol 15: 138

19. Shepherd FA, Rodrigues Pereira J, Ciuleanu T et al. (2005) Erlotinib in Previously Treated Non-SmallCell Lung Cancer. N Engl J Med 353: 123

20. Simpson AJ, Caballero OL, Jungbluth A et al. (2000) Cancer/testis antigens, gametogenesis and cancer. Nat Rev Cancer 5: 615

21. Slamon DJ, Leyland-Jones B, Shak S et al. (2001) Use of chemotherapy plus a monoclonal antibody against HER2 for metastatic breast cancer that overexpresses HER2. N Engl J Med 344: 783

22. Talpaz M, Shah NP, Kantarjian H et al. (2006) Dasatinib in imatinib resistant Philadelphia chromosome-positive leukemias. N Engl J Med 354: 2531

23. van der Bruggen $P$, Traversari $C$, Chomez $P$ et al. (1991) A gene encoding an antigen recognized by cytolytic T lymphocytes on a human melanoma. Science 13(254): 1643
24. Varga Z, Theurillat JP, Filonenko V et al. (2006) Preferential nuclear and cytoplasmic NY-BR-1 protein expression in primary breast cancer and lymph node metastases. Clin Cancer Res 12: 2745 